\title{
Quantification of the Resolved Phase Change in Reconstructed Electron Exit Waves of Gold [110] in Different Electron Microscopes
}

\author{
J.R. Jinschek*, C. Kisielowski*, M. Lentzen**, K. Urban** \\ * National Center for Electron Microscopy (NCEM), Lawrence Berkele \\ (LBNL), 1 Cyclotron Road MS 72, Berkeley, CA 94720, U.S.A. \\ y National Laboratory \\ ** Forschungszentrum Jülich GmbH, D-52425 Jülich, Germany
}

Today material science benefits from high -resolution transmission electron microscopy (HRTEM) with a resolution tha $t$ extends to the information limit of field emission microscopes into the sub Ångstrøm region. Procedures to retrieve this resolution involve a reconstruction of the complex electron exit-plane wave function by holographic recording techniques such as from focal series of HRTEM images [1] or from single off -axis electron holograms [2]. In this contribution we compare the recovered exit -plane phase images of electron microscopes with different contrast transfer functions (CTF) and consequentially different i nformation limits. We tested a JEOL 3010 (LaB ${ }_{6}$ ), a CM200 FEG, a CM200 FEG with C s corrector [3], and a CM300 FEG/UT (OAM) [4] by taking images from the same sample of gold [110].

The electron exit wave function was reconstructed from HRTEM lattice images of focal series by the PAM/MAL-algorithm of the Philips/Brite-Euram software package [5]. An example is shown in Fig. 1. Theory predicts that the intensity in reconstructed exit wave phase images oscillates with sample thickness showing maxima of magnitude $2 \pi$. Such oscillations are readily observable on wedge shaped samples (see line scan in Fig. 2). However, even a reconstruction of the electron exit wave from simulated lattice images already reveals a substantial decrease of the phase magnitude at a maximum that strongly depends on the resolution and the contrast transfer function of the microscope under consideration. This effect is caused by distribution of phase information across an area determined by a limited resolution. It can be exploited to assign a figure of merit to a particular electron microscope by quantification of the maximum phase signal that can be retrieved in an experiment on wedge shaped samples and compared to the recorded noise level. This is depicted in Fig. 3. The good figure of mer it of the $\mathrm{C}_{\mathrm{S}}$ corrected microscope is remarkable since it reaches values almost as large as those from the OAM. Consequently, the microscope allows to record crystal and defect structures with an excellent signal to noise ratio as shown in Fig. 4. In compar ison with theory, a further decrease of phase values is observed experimentally that may be related to the "Stobbs factor" and typical phase values do not exceed $1 \mathrm{rad}$ at noise levels smaller than $20 \mathrm{mrad}$. Due to the large scattering power of gold ( $\mathrm{Au}$, ato mic number $\mathrm{Z}=79$ ) a phase change per single gold atom can be determined. With this value one can extract the number of gold atoms in individual atomic columns and determine sensitivity limits that will be given [6].

References

[1] D. Van Dyck, et al., Optik 93 (1993) 103.

[2] H. Lichte, Advances in Optical and Electron Microscopy 12 (1991) 25.

[3] M. Haider, et al., Ultramicroscopy 75 (1998) 53.

[4] C. Kisielowski, et al., Ultramicroscopy 89 (2001) 243 and M.A. O'Keefe, et al., Ultramicroscopy 89 (2001) 215.

[5] W.M.J. Coene, et al., Ultramicroscopy 64 (1996) 109 and A. Thust, et al., Ultramicroscopy 64 (1996) 211. 
[6] The project was sponsored by the Director, Office of Science, Office of Basic Energy Sciences, of the U.S. Department of Energy under Contract No. DE-AC03-76SF00098. J.R. Jinschek was supported by a Feodor-Lynen-Fellowship of the Alexander-von-Humboldt Foundation (Bonn/Germany, 'http://Www.avh.dę).

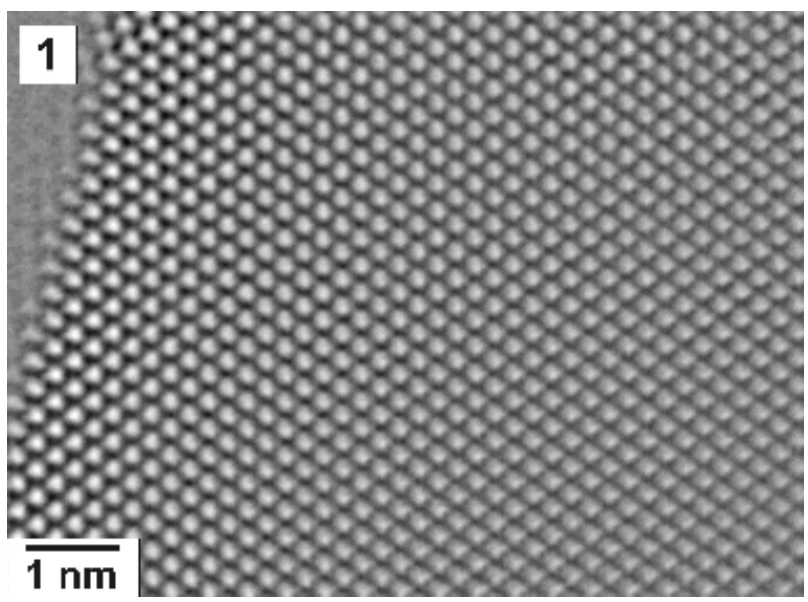

FIG. 1. Phase image of a gold wedge shaped sample after exit wave reconstru ction in the CM300 FEG (OAM) [4].

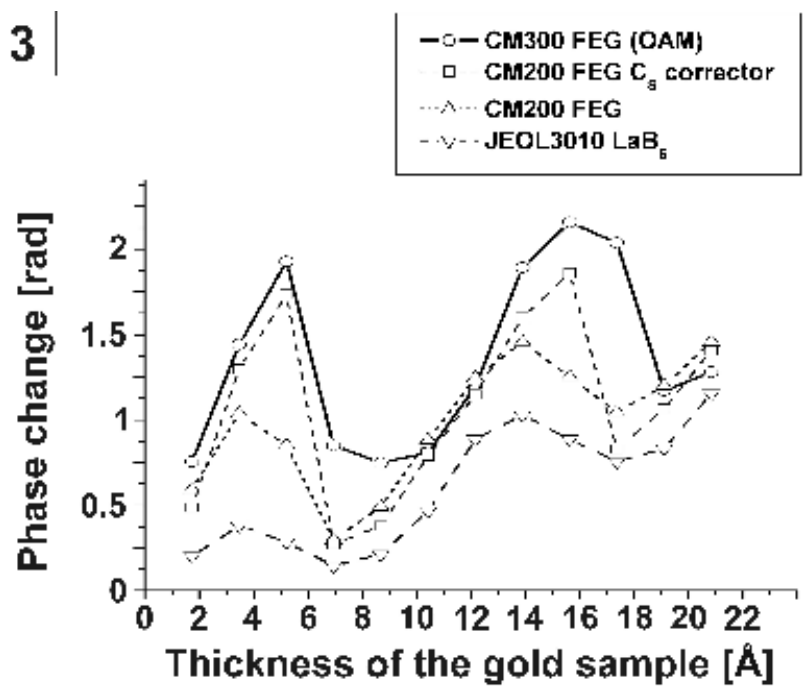

FIG. 3. Calculated phase oscillations at a gold wedge in different utilized electron microscopes.

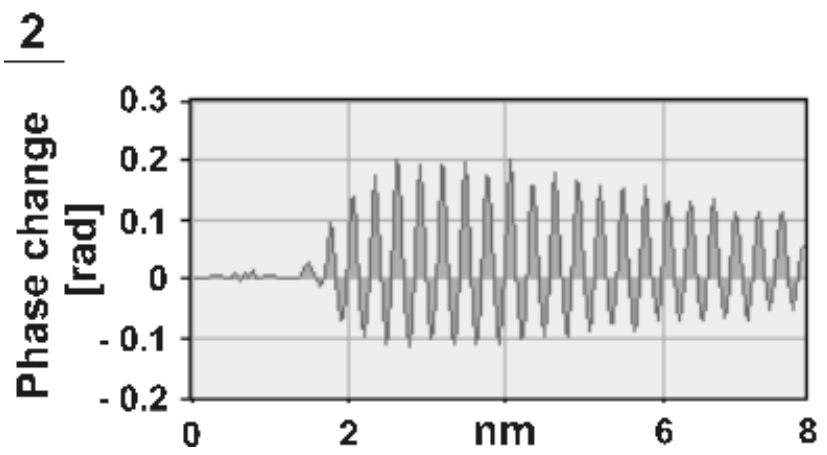

FIG. 2. Phase oscillations on the wedge shaped sample (line scan marked in Fig. 4).

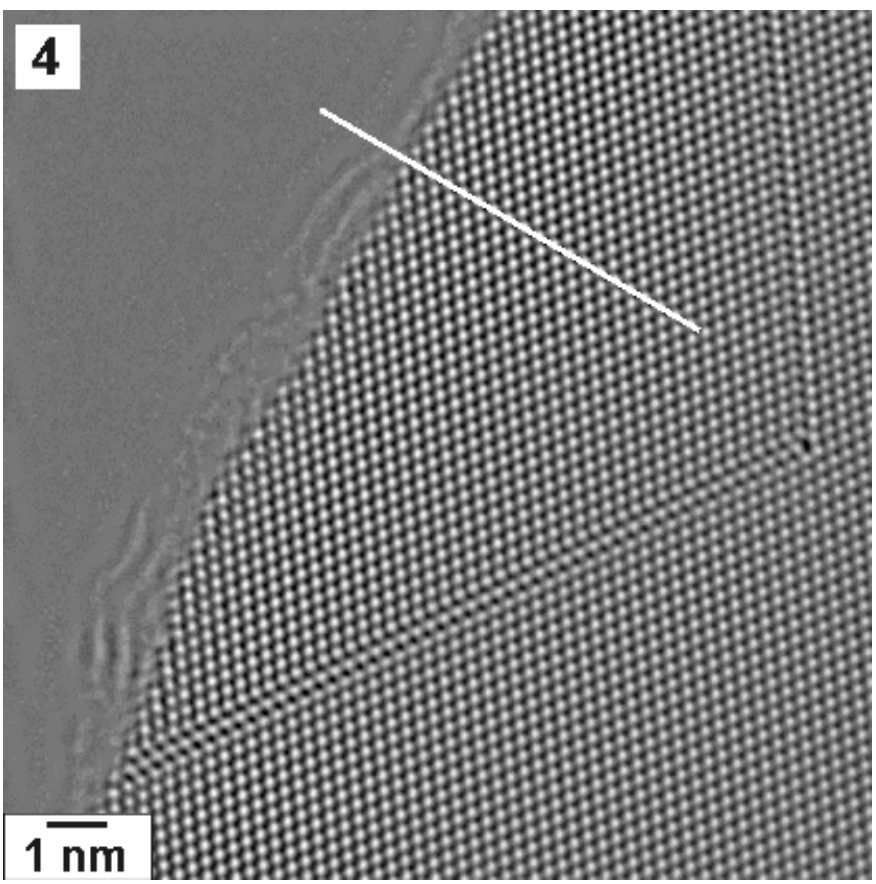

FIG. 4. Phase im age of a gold wedge shaped sample after exit wave reconstruction in the $\mathrm{C} s$ corrected CM200 FEG [3]. 